\title{
Relay Selection and Power Allocation in Amplify-and-Forward Cognitive Radio Systems Based on Spectrum Sharing
}

\author{
Daqian Zhao, Zhizhong Zhang, Fang Cheng \\ School of Communication and Information Engineering, Chongqing University of Posts and Telecommunications, Chongqing, China \\ Email: 359411295@qq.com, zhangzz@cqupt.edu.cn, chengfang@cqupt.edu.cn
}

Received July, 2013

\begin{abstract}
In this paper, we consider a spectrum sharing scheme that is a joint optimization of relay selection and power allocation at the secondary transmitter, which aims to achieve the maximum possible throughput for the secondary user. This paper considers the scenario where the primary user is incapable of supporting its target signal-to-noise ratio (SNR). More especially, the secondary transmitter tries to assist the primary user with achieving its target SNR by cooperative amplify-and-forward (AF) relaying with two-phase. By exhaustive search for all candidate secondary transmitters, an optimal secondary transmitter can be selected, which not only can satisfy the primary user's target SNR, but also maximize the secondary user's throughput. The optimal secondary transmitter acts as a relay for the primary user by allocating a part of its power to amplify-and-forward the primary signal over the primary user's licensed spectrum bands. At the same time, as a reward, the optimal secondary transmitter uses the remaining power to transmit its own signal over the remaining licensed spectrum bands. Thus, the secondary user obtains the spectrum access opportunities. Besides, there is no interference between the primary user and the secondary user. We study the joint optimization of relay selection and power allocation such that the secondary user's throughput is maximized on the condition that it satisfies the primary user's target SNR. From the simulation, it is shown that the joint optimization of relay selection and power allocation provides a significant throughput gain compared with random relay selection with optimal power allocation (OPA) and random relay selection with water-filling power allocation (WPA). Moreover, the simulation results also shown that our spectrum sharing scheme obtains the win-win solution for the primary system and the secondary system.
\end{abstract}

Keywords: Spectrum Sharing; Cooperative Communication; Relay Selection; Power Allocation; Throughput; Maximum Ratio Combining; Cognitive Radio Systems

\section{Introduction}

Currently, the fixed spectrum access (FSA) policy has traditionally been adopted by spectrum regulators, which assigns each piece of spectrum with certain bandwidth to one or more dedicated users. By doing so, only the assigned (licensed) users have the right to exploit the allocated spectrum, and other users are not allowed to use it, regardless of whether the licensed users are using it or not. Recent studies on the actual spectrum utilization measurements have revealed that a large portion of the licensed spectrum experiences low utilization [1-3]. However, cognitive radio (CR) is an agile spectrum access/sharing technology that allows unlicensed (secondary) systems to operate in licensed frequency bands without causing harmful interference to licensed (primary) systems, and thus spectrum utilization can be significantly improved [4-6]. Thus, CR is widely regarded as one of the most promising technologies for future wireless communications.

Cooperative relay communication can improve power efficiency in the wireless networks by increasing the spatial diversity. By the use of relays, the transmitted power from the source terminal can be significantly reduced [7-9].

The literature [10] proposed an opportunistic spectrum sharing protocol that exploited the scenario where the primary system was incapable of supporting its target transmission rate. Specifically, the secondary system tried to assist the primary system with achieving its target rate via two-phase cooperative OFDM relaying. However, the literature [10] didn't study the problem of relay selection in a multi-relay secondary system, so the outage was more likely occur at the primary system. If the outage was occurred at the primary system, it wasn't the win-win solution for the primary system and the secondary system. The literature [10] used the dual decompo- 
sition method and the authors was derived the closed-form solutions for the set of subcarriers, subcarrier pairing and power allocation, so the algorithm was complex. In our paper, we study the problem of relay selection in a multi-relay secondary system so that the outage can't easily occur at the primary system. Furthermore, the algorithm complexity in our paper is straightforward compared with the literature [10] and the simulation results also shown that our spectrum sharing scheme is better than [10].

In [11], the authors studied the problem of joint relay selection and power allocation at the source and relay the nodes in a CR system in which nodes were allowed to amplify-and-forward cooperate with each other. However, the literature [11] used the opportunistic spectrum access (OSA) model: the CR user carried out spectrum sensing to detect spectrum holes [12], so it presented a very high demand for the spectrum sensing accuracy when the primary system exist, and it was not easily to realize. In addition, the literature [11] had set up the interference threshold to protect the primary user, so the throughput was limited. In our paper, we don't need set up the interference threshold to protect the primary user. On the contrary, we assist the primary user with achieving its target SNR, as a reward, the secondary user can access the primary user's licensed spectrum bands.

In this paper, we study the joint optimization of relay selection and power allocation such that the secondary user's throughput is maximized on the condition that it satisfies the primary user's target SNR. By exhaustive search for all candidate secondary transmitters, an optimal secondary transmitter can be selected, which not only can satisfy the primary user's target SNR, but also maximize the secondary user's throughput. Since the optimal secondary transmitter uses different licensed spectrum bands to amplify-and-forward primary signal and to transmit its own signal, so there is no interference between the primary user and the secondary user. From the simulation, it is shown that the joint optimization of relay selection and power allocation provides a significant throughput gain compared with OPA and WPA. Moreover, the simulation results also shown that our spectrum sharing scheme obtains the win-win solution for the primary system and the secondary system.

The rest of this paper is organized as follows. In Section 2, we introduce the system model. System performance is analyzed in Section 3. The joint optimization of resource allocation is presented in Section 4. Simulation results are shown in Section 5. Finally, this paper is concluded in Section 6.

\section{System Description}

We consider cooperative cognitive radio systems as shown in Figure 1. The primary system, comprising of a primary user base station (PUBS) and a primary user receiver (PUR), supports the relaying functionality and has the license to operate in some spectrum bands. We assume that the primary system has multiple licensed spectrum bands as shown in Figure 2, and PUR can keep track of the SNR of the PUBS $\rightarrow$ PUR link. The secondary system, comprising of $n$ secondary user transmitters $\left(S U T_{i}, i=1 . . n\right)$ and a secondary user receiver (SUR), can only operate in licensed spectrum bands by using the scenario where the PUR is incapable of supporting its target SNR (e.g., the SNR of the PUBS $\rightarrow$ PUR link is below a threshold due to path loss, shadowing, moving, or interference). Specifically, the secondary transmitter tries to assist the primary user with achieving its target SNR by cooperative AF relaying with two-phase. The scenario provides an opportunity for the secondary user to access the licensed spectrum bands of the primary system. Since the optimal secondary transmitter uses different licensed spectrum bands to amplify-and-forward primary signal and to transmit its own signal, so there is no interference between the primary user and the secondary user. We assume that the secondary system is able to emulate system parameters of the primary system. For the sake of simplicity, we assume that the $i^{\text {th }}$ relay $\left(S U T_{i}\right.$ ) has been selected as the optimal relay. The problem of relay selection will be addressed in Section 4 .

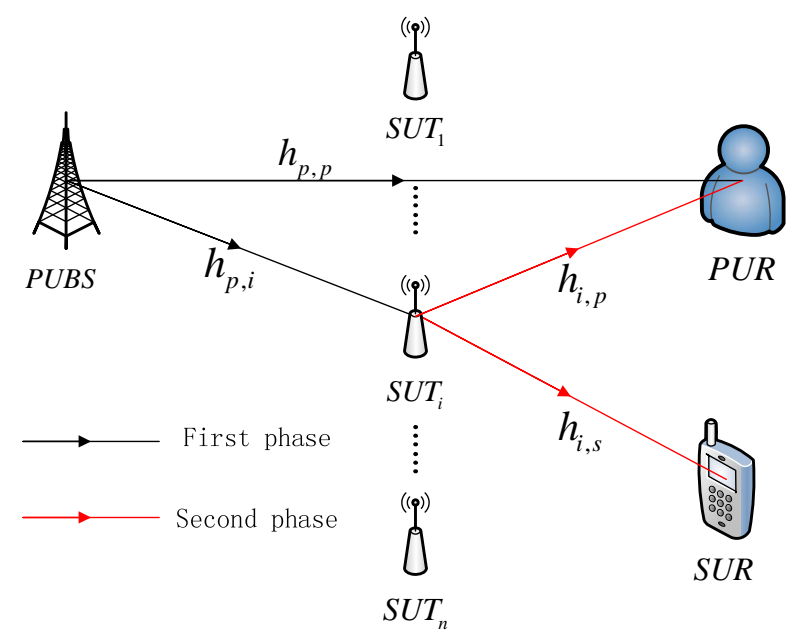

Figure 1. System model.

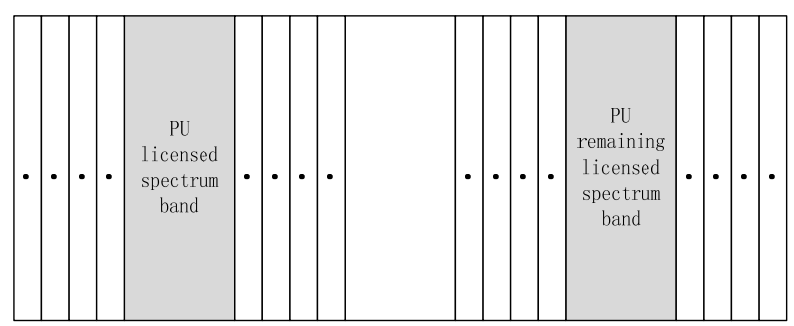

Figure 2. PU's licensed spectrum bands. 
Both the primary system and the secondary system experience independent and frequency-selective Rayleigh fading. The channel gains of the PUBS $\rightarrow P U R$, $P U B S \rightarrow S U T_{i}, S U T_{i} \rightarrow P U R, S U T_{i} \rightarrow S U R$ links are denoted as $h_{p, p}, h_{p, i}, h_{i, p}, h_{i, s}$, respectively. We assume that these channel state information (CSI) are available at prior to transmission. We consider slow fading where the channel gains remain constant over the one-phase. We assume that $n_{p, p}, n_{p, i}, n_{i, p}, n_{i, s}$ are independent additive white Gaussian noises with zero mean and variance $N_{0}$. Let $X_{p}$ and $X_{s}$ denote the signal to be transmitted to PUR and SUR, respectively, which have unit power. The PUBS and $S U T_{i}, i=1 \ldots n$ each have a sum transmit power constraint, denoted as $P_{\max }^{P U B S}$ and $P_{\max }^{S U T_{i}}, i=1 \ldots n$.

\section{Analysis of Performance}

In the first phase, as shown by the black solid arrows in Figure 1, PUBS transmits the signal $X_{p}$ while PUR and $S U T_{i}$ listen. The signal received at PUR in the first phase is given by

$$
y_{p, p}=\sqrt{P_{p}} h_{p, p} X_{p}+n_{p, p}
$$

where $P_{p}$ is the power transmitted from PUBS to PUR and PUBS to $S U T_{i}$. PUBS uses the maximum transmission power $P_{\max }^{P U B S}$ to transmit the primary signal, namely as $P_{p}=P_{\max }^{\text {PUBS }}$. From equation (1), the instantaneous SNR of PUR in the first phase can be written as

$$
\gamma_{1}=\frac{P_{p}\left|h_{p, p}\right|^{2}}{N_{0}}
$$

Similarly, the signal received at $S U T_{i}$ is given by

$$
y_{p, i}=\sqrt{P_{p}} h_{p, i} X_{p}+n_{p, i}
$$

In the second phase, as shown by the red solid arrows in Figure 1, PUBS remains silent while $S U T_{i}$ amplifyand-forward primary signal over the primary user's licensed spectrum bands as shown in Figure 2. The signal received at PUR in the second phase can be written as

$$
y_{i, p}=\beta_{i} h_{i, p} y_{p, i}+n_{i, p}
$$

where $\beta_{i}$ is the amplification factor and is defined as

$$
\beta_{i}=\frac{\sqrt{P_{s, p}}}{\sqrt{P_{p}\left|h_{p, i}\right|^{2}+N_{0}}}
$$

where $P_{s, p}$ is the power transmitted from $S U T_{i}$ to PUR. By replacing equation (3) and equation (5) into equation (4), we have

$$
y_{i, p}=\frac{\sqrt{P_{p} P_{s, p}} h_{i, p} h_{p, i}}{\sqrt{P_{p}\left|h_{p, i}\right|^{2}+N_{0}}} X_{p}+n_{i, p}^{\prime}
$$

where $n_{i, p}^{\prime}=\frac{\sqrt{P_{s, p}} h_{i, p}}{\sqrt{P_{p}\left|h_{p, i}\right|^{2}+N_{0}}} n_{p, i}+n_{i, p}$. Since $n_{p, i}$ and $n_{i, p}$ are independent additive white Gaussian noises, $n_{i, p}^{\prime}$ is also Gaussian with zero mean and variance

$$
N_{0}^{\prime}=\left(\frac{P_{s, p}\left|h_{i, p}\right|^{2}}{P_{p}\left|h_{p, i}\right|^{2}+N_{0}}+1\right) N_{0}
$$

From equation (6) and equation (7), the instantaneous SNR at PUR in the second phase can be written as

$$
\gamma_{2}=\frac{P_{p} P_{s, p}\left|h_{i, p}\right|^{2}\left|h_{p, i}\right|^{2}}{\left(P_{p}\left|h_{p, i}\right|^{2}+P_{s, p}\left|h_{i, p}\right|^{2}+N_{0}\right) N_{0}}
$$

It has been shown that applying the maximum ratio combining at PUR in an AF relay system maximized the SNR [13]. Therefore, we use the maximum ratio combining (MRC) at PUR to combine the two received signals from the first phase and the second phase. We assume that PUR has all the CSIs. As the instantaneous SNR at the output of combiner equals the sum of the SNRs of the incoming signals [13], we can write the instantaneous SNR $\gamma_{p}$ after applying the MRC as

$$
\gamma_{p}=\gamma_{1}+\gamma_{2}
$$

where $\gamma_{1}$ and $\gamma_{2}$ are obtained from equation (2) and equation (8), respectively. Since $S U T_{i}$ must be assist PUR with achieving its target SNR by acting as an AF relay, the PUR's target SNR constraint as follows

$$
\gamma_{p} \geq \gamma_{T}
$$

where $\gamma_{T}$ is the PUR's target SNR.

In the second phase, $S U T_{i}$ also uses its remaining power to transmit its own signal over the remaining licensed spectrum bands on the condition that it satisfies the PUR's target SNR as shown in Figure 2. The signal received at SUR is given by

$$
y_{i, s}=\sqrt{P_{s, s}} h_{i, s} X_{s}+n_{i, s}
$$

where $P_{s, s}$ is the power transmitted from $S U T_{i}$ to SUR. From equation (11), the instantaneous SNR at SUR in the second phase can be written as

$$
\gamma_{s}=\frac{P_{s, s}\left|h_{i, s}\right|^{2}}{N_{0}}
$$

Therefore, assuming that the $i^{\text {th }}$ relay has been selected, we can write the instantaneous throughput of SUR as

$$
T_{i}\left(P_{s, s}\right)=\log _{2}\left(1+\gamma_{s}\right)
$$

Since $P_{s, p}$ and $P_{s, s}$ are the transmit power from $S U T_{i}$, this two transmit power must be satisfy the fol- 
lowing constraint

$$
P_{s, p}+P_{s, s} \leq P_{\max }^{S U T_{i}}
$$

In the next section, we solve the joint optimization of resource allocation problem to maximize the instantaneous throughput of SUR in equation (13).

\section{Resource Allocation}

In this section, we solve the joint optimization of relay selection and power allocation at the secondary transmitter to achieve the maximum possible throughput for the SUR while guaranteeing the PUR to achieve its target SNR. The optimal power allocation at the secondary transmitter to maximize the throughput for the SUR is calculated. Then, through an exhaustive search for all candidate secondary transmitters, an optimal secondary transmitter can be selected, which not only can satisfy the primary user's target SNR, but also maximize the secondary user's throughput as defined in equation (13). Therefore, in the following, we set up the optimization problem to find the optimum set of transmit powers $P_{s, p}$ and $P_{s, s}$ for the secondary transmitter. The power allocation problem for all candidate secondary transmitters can be formulated as the following optimization

$$
\left(P_{s, s, i}^{*}\right)=\arg \max _{P_{s, s}} T_{i}\left(P_{s, s}\right)
$$

subject to

$$
\begin{aligned}
& \gamma_{p} \geq \gamma_{T} \\
& P_{s, p}+P_{s, s} \leq P_{\max }^{S U T_{i}} \\
& P_{s, p}>0, P_{s, s}>0
\end{aligned}
$$

where $P_{s, s, i}^{*}$ is the optimal values of $P_{s, s}$ for the $i^{\text {th }}$ secondary transmitter. The optimal relay selection can be formulated as an exhaustive search for all candidate secondary transmitters

$$
\hat{i}=\arg \max _{i} T_{i}\left(P_{s, s, i}^{*}\right)
$$

where $i$ ranges from 1 to $n$ and $i$ represents the optimal secondary transmitter. From equation (10) we can obtain

$$
P_{s, p} \geq \frac{\left(\gamma_{T}-\gamma_{1}\right)\left(P_{p}\left|h_{p, i}\right|^{2} N_{0}+N_{0}^{2}\right)}{P_{p}\left|h_{i, p}\right|^{2}\left|h_{p, i}\right|^{2}-\left(\gamma_{T}-\gamma_{1}\right)\left|h_{i, p}\right|^{2} N_{0}}
$$

According to our spectrum sharing scheme, as long as the optimal secondary transmitter can satisfy the PUR's target SNR, it can access the remaining licensed spectrum bands instead of exceeding the PUR's target SNR. Therefore, the equation (17) can be rewritten as

$$
P_{s, p}=\frac{\left(\gamma_{T}-\gamma_{1}\right)\left(P_{p}\left|h_{p, i}\right|^{2} N_{0}+N_{0}^{2}\right)}{P_{p}\left|h_{i, p}\right|^{2}\left|h_{p, i}\right|^{2}-\left(\gamma_{T}-\gamma_{1}\right)\left|h_{i, p}\right|^{2} N_{0}}
$$

From equation (14), we know that the power allocation is optimal when $P_{s, p}+P_{s, s}=P_{\max }^{S U T_{i}}$, namely as

$$
P_{s, s}=P_{\max }^{S U T_{i}}-P_{s, p}
$$

By replacing equation (18) into equation (19), the optimal power allocation $P_{s, s, i}^{*}$ can be written as

$$
P_{s, s, i}^{*}=P_{\max }^{S U T_{i}}-\frac{\left(\gamma_{T}-\gamma_{1}\right)\left(P_{p}\left|h_{p, i}\right|^{2} N_{0}+N_{0}^{2}\right)}{P_{p}\left|h_{i, p}\right|^{2}\left|h_{p, i}\right|^{2}-\left(\gamma_{T}-\gamma_{1}\right)\left|h_{i, p}\right|^{2} N_{0}}
$$

The optimal secondary transmitter is then found by an exhaustive search for all candidate secondary transmitters and finding the optimal secondary transmitter that maximizes the throughput using equation (16).

\section{Simulation Results}

In this section, the simulation results are presented to demonstrate the performance of our spectrum sharing scheme in terms of SUR throughout.

We consider cooperative cognitive radio systems with $n$ secondary transmitters operating in AF mode as shown in Figure 1. All the channels are assumed to be Rayleigh fading with unity bandwidth. We assume that $P_{\max }^{\text {PUBS }}=10 \mathrm{~W}, N_{0}=0.1 \mathrm{~W} / \mathrm{Hz}$.

First, we simulate that SUR throughput versus PUR target SNR, assuming that $P_{\max }^{S U T_{i}}=10 \mathrm{~W}, i=1 \ldots n$. The number of the candidate secondary transmitters are fixed at $n=10$, which have already satisfied the PUR's target SNR. We apply our spectrum sharing scheme and simulate that SUR throughput versus PUR target SNR in Figure 3. We have also simulated the cases of random relay selection with optimal power allocation (OPA) and random relay selection with water-filling power allocation (WPA). Similarly, both of two algorithms have already satisfied the PUR's target SNR. Note in the WPA that the power allocation at PUBS and $S U T_{i}$ are predetermined by the water-filling algorithm based on the $P U B S \rightarrow$ PUR channel and the $S U T_{i} \rightarrow$ SUR channel,

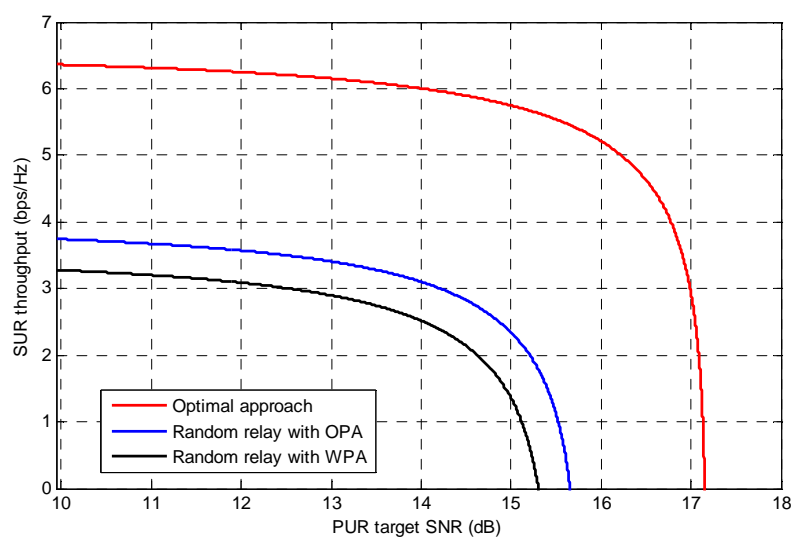

Figure 3. SUR throughput versus PUR target SNR. 
respectively. From Figure 3, we see that the SUR throughput increases significantly when our spectrum sharing scheme for relay selection and power allocation is applied compared with the OPA and the WPA. When the PUR's target $S N R \leq 9.95 d B$, it means that the PUR no need to cooperate and the SUR throughput is the maximum equal $6.3626 \mathrm{bps} / \mathrm{Hz}$. However, the optimal secondary transmitter can't access the PU's licensed spectrum bands, so it's a peak for the SUR throughput. When the PUR's target $S N R>9.95 d B$, the PUR seeks to cooperate and the optimal secondary transmitter assists the PUR with achieving its target SNR by acting as an AF relaying with two-phase. Then, as a reward, the optimal secondary transmitter can access the remaining licensed spectrum bands. With the PUR target SNR increases, the optimal secondary transmitter needs allocate more power to assist the PUR with achieving its target SNR and thus leading to a lower SUR throughput. Applying our optimal approach the SUR throughput gains of $2.6351 \mathrm{bps} / \mathrm{Hz}$ and $3.1046 \mathrm{bps} / \mathrm{Hz}$ compared with the OPA and the WPA are obtained, respectively, at the PUR's target $S N R=12 d B$. When $S N R>17 d B$ in the optimal approach; $S N R>15 d B$ in the OPA and the WPA, the SUR throughput rapid decline. This is because the optimal secondary transmitter allocates more power to amplify-and-forward the primary signal, so the SUR throughput decreases rapidly.

Figure 4 shows that SUR throughput versus $P_{\max }^{S U T_{i}}$ compared with the OPA and the WPA while the PUR's target SNR is fixed at $12 \mathrm{~dB}$ and the number of the candidate secondary transmitters are fixed at $n=10$. When $P_{\max }^{S U T_{i}}=5 \mathrm{~W}$, the optimal approach provides 2.6338 $\mathrm{bps} / \mathrm{Hz}$ and $3.0904 \mathrm{bps} / \mathrm{Hz}$ throughput gains compared with the OPA and the WPA, respectively. Because a part of the transmit power is allocated to amplify-and-forward the primary signal, so the curve didn't start from zero. From the Figure 4, we also can see that the OPA and the WPA need allocate more power to amplify-and-forward the primary signal compared with optimal approach.

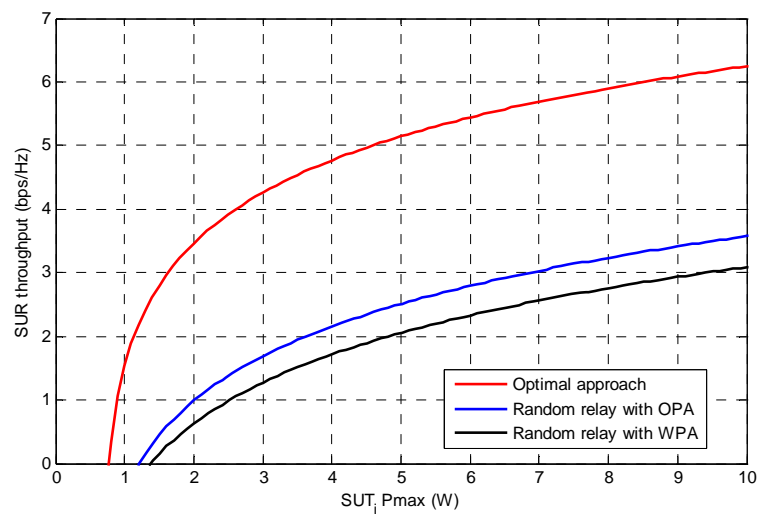

Figure 4. SUR throughput versus $P_{\max }^{S U T_{i}}$.



Figure 5. SUR throughput versus the number of candidate $S U T_{i}$.

Figure 5 shows that SUR throughput versus the number of candidate $S U T_{i}$ compared with the OPA and the WPA while the PUR's target $S N R=12 d B$ and $P_{\max }^{S U T_{i}}=10 \mathrm{~W}, i=1 \ldots n$. As seen, the optimal approach provides an appealing throughput increase by increasing the number of candidate $S U T_{i}$ while the OPA and the WPA do not provide any throughput increase. This is because the optimal secondary transmitter is selected randomly in the OPA and the WPA.

\section{Conclusions}

In this paper, we consider a spectrum sharing scheme that is a joint optimization of relay selection and power allocation at the secondary transmitter, which aims to achieve the maximum possible throughput for the secondary user. This paper considers the scenario where the primary user is incapable of supporting its target SNR. Specifically, the secondary transmitter tries to assist the primary user with achieving its target SNR by allocating a part of its power to amplify-and-forward the primary signal over the primary user's licensed spectrum bands. At the same time, as a reward, the secondary transmitter uses the remaining power to transmit its own signal over the remaining licensed spectrum bands. By exhaustive search for all candidate secondary transmitters, an optimal secondary transmitter can be selected, which not only can satisfy the primary user's target SNR, but also maximize the secondary user's throughput. We study the joint optimization of relay selection and power allocation such that the secondary user's throughput is maximized on the condition that it satisfies the primary user's target SNR. Simulation results confirmed the efficiency of this spectrum sharing scheme and it benefits to both the primary system and the secondary system.

\section{Acknowledgements}

This work is partially supported by National Major Sci- 
ence and Technology Special Project of China (2012ZX03005008, 2012ZX03005002-005), Chongqing Municipal Education Commission Science and Technology Research Project (KJ130513), Chongqing Basic and Cutting edge Project (cstc2013jcyjA40020), 2011 Chongqing Universities outstanding achievement transformation funded project, 2010 Chongqing Municipal Intellectual Property special funds.

\section{REFERENCES}

[1] P. Kolodzy and I. Avoidance, "Spectrum Policy Task force,” Federal Commun. Comm., Washington, DC, Rep. ET Docket, 2002 (02-135).

[2] M. H. Islam, C. L. Koh, S. W. Oh, et al., Spectrum Survey in Singapore: Occupancy Measurements and Analyses[C]//Cognitive Radio Oriented Wireless Networks and Communications, 2008. Crown Com 2008. 3rd International Conference on. IEEE, 2008, pp. 1-7.

[3] D. Datla, A. M. Wyglinski and G. J. Minden, "A Spectrum Surveying Framework for Dynamic Spectrum Access Networks," Vehicular Technology, IEEE Transactions on, Vol. 58, No. 8, 2009, pp. 4158-4168. doi:10.1109/TVT.2009.2021601

[4] III. J. Mitola and Jr. G. Q. Maguire, “Cognitive Radio: Making Software Radios More Personal,” Personal Communications, IEEE, 1999, Vol. 6, No. 4, pp. 13-18. doi:10.1109/98.788210

[5] S. Haykin, "Cognitive Radio: Brain-empowered Wireless Communications," Selected Areas in Communications, IEEE Journal on, Vol. 23, No. 2, 2005, pp. 201-220. doi:10.1109/JSAC.2004.839380

[6] A. Jovicic and P. Viswanath, "Cognitive Radio: An Information-theoretic Perspective," Information Theory,
IEEE Transactions on, Vol. 55, No. 9, 2009, pp. 3945-3958. doi:10.1109/TIT.2009.2025539

[7] C. H. Chen, C. L. Wang and C. T. Chen, "A Resource Allocation Scheme for Cooperative Multiuser OFDM-Based Cognitive Radio Systems,” Communications, IEEE Transactions on, Vol. 59, No. 11, 2011, pp. 3204-3215. doi:10.1109/TCOMM.2011.092011.100197

[8] M. Shaat and F. Bader, “Asymptotically Optimal Resource Allocation in OFDM-based Cognitive Networks with Multiple Relays,” Wireless Communications, IEEE Transactions on, Vol. 11, No. 3, 2012, pp. 892-897. doi:10.1109/TWC.2012.011012.110880

[9] L. Li, X. Zhou, H. Xu, et al., "Simplified Relay Selection and Power Allocation in Cooperative Cognitive Radio Systems," Wireless Communications, IEEE Transactions on, Vol. 10, No. 1, 2011, pp. 33-36. doi:10.1109/TWC.2010.101810.100311

[10] W. D. Lu, Y. Gong, S. H. Ting, et al., "Cooperative OFDM Relaying for Opportunistic Spectrum Sharing: Protocol Design and Resource Allocation," Wireless Communications, IEEE Transactions on, 2012, Vol. 11, No. 6, pp. 2126-2135. doi:10.1109/TWC.2012.032812.110524

[11] K. R. Budhathoki, M. Maleki and H. R. Bahrami, "Relay Selection and Power Allocation in Amplify-and-forward Cognitive Radio Systems[C]//Computing, Networking and Communications (ICNC), 2013 International Conference on. IEEE, 2013, pp. 686-690.

[12] Y. C. Liang, K. C. Chen, G. Y. Li, et al., "Cognitive Radio Networking and Communications: An Overview," Vehicular Technology, IEEE Transactions on, 2011, Vol. 60, No. 7, pp. 3386-3407. doi:10.1109/TVT.2011.2158673

[13] K. J. R. Liu, "Cooperative Communications and Networking,” Cambridge University Press, 2009. 